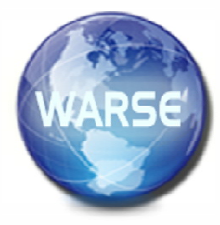

\title{
Modularity Based Community Detection in Dynamic Social Networks
}

\author{
Bipin Gupta $^{1}$, Dr. Ashutosh Singh ${ }^{2}$, Dr. A.K. Shankhwar ${ }^{3}$ \\ ${ }^{1}$ Department of Electronics Engineering H.B.T.U, India, Bp2242990@ gmail.com \\ ${ }^{2}$ Department of Electronics Engineering H.B.T.U, India, ashu@ gmail.com \\ ${ }^{3}$ Department of Electronics Engineering H.B.T.U, India, sak_neeraj@yahoo.com
}

\begin{abstract}
Regular change is one of the features of network observed while analyzing dynamic social networks. But it is not easy to find community detection on real world dynamic social network. Dynamic social network generators are used insertion/deletion for node/edge and merge/split of community. In this paper, a method for community detection in a dynamic social network is proposed. Initially we take a static network, by moving a clique from one community to another community and flipping edge method in static network. By repeating this step, we get a dynamic network with improved modularity at every time step. So this method is used for community detection in dynamic network. An experiment is conducted to study changes in various network parameters like number of node, clustering coefficient, average distance between nodes etc.
\end{abstract}

Key words: clustering coefficient, dynamic network, modularity, social network.

\section{INTRODUCTION}

In social network, nodes represented as core and edges of network represent relationship between nodes. In study of evolution of dynamic network we get a deeper insight into their structure and characteristics. Social network generators are divided into static and dynamic type. These generators add/remove nodes, edge. A generator which is based on modularity in both static and dynamic is used for community detection.

In this paper, which extends the work by [01] we purpose a method, which improves the modularity value and parameter of network. In DSNG-M (Dynamic social network generator -based on modularity) [02] only consider edge evolution and TESN-M (Time evolving social network - based on modularity) [01] node and edge evolution with decrease modularity and clustering coefficient.

We extend this work with improved parameter of network. Modularity at each time step is calculated according to common community partition. We take a clique and move from one community to other community and then calculate modularity. We then check limiting condition and apply flip operation and calculate modularity if difference between calculated modularity is smaller than the difference between modularity before flipping and expected modularity then we accept flipping otherwise flipping is accepted with decrease probability over time.

In node evolution, we can control that inserted node belong to which community. When node deleted then node disappear from the whole network. This process is closer to real world.[14] The result of proposed method is given in section IV. Modularity of most real world social network is (0.3-0.7). [12] Hence in proposed method we set a threshold value of expected modularity in the range (0.3-0.7). In addition, we propose that the modularity of dynamic synthetic network can be used as the performance base line of community detection algorithm in dynamic social network.

Overall contribution of this paper is given below.

1. We propose a method which is the extended version of TESN-M [01]. Network generated by proposed method can simulate the property of real time evolving social network.

2. We propose that modularity of dynamic network is base line for the performance of community detection algorithm.

Rest of the paper is organized as follows. Section II describes related work and the detail of proposed work is explained in section III. Section IV presents the experimental result and analysis while the conclusion is given section $\mathrm{V}$.

\section{RELATED WORK}

In this paper, we briefly introduce dynamic network generator. Community detection based on static and dynamic network. After this we describe the concept of modularity because it is main feature of generator.

\subsection{Static community detection}

These are many effective no of algorithm is given. [11] For example Leo et al [03] proposed community detection by fuzzy relation (CDFR) in which to find out NGC node (nearest node with greater centrality). Shang et al [04] 
proposed a modularity integration method to detect large network. Wu et al [05] proposed a method to detect community of network by clustering dynamic of network.

\subsection{Dynamic social network}

\subsubsection{Dynamic social network generator [08] [13]}

This is classified in mainly two parts.

(i) Basic evolution property

(ii) Structural evolution of real network.

Few generators are used to simulate structural evolution of real world dynamic network.

In this paper, our proposed method used original social network with labels and expected modularity.

\subsection{Modularity}

\subsubsection{Static modularity}

First modularity was proposed by Newman. It plays an important role quality of community detection. [06]

$$
\begin{array}{ll}
\mathbf{Q}=\sum_{\mathbf{s}}\left(\frac{\mathbf{K}_{\mathbf{s}}^{\text {in }}}{2 \mathbf{M}}-\left(\frac{\mathbf{K}_{\mathbf{s}}}{2 \mathbf{M}}\right)^{2}\right) \ldots(\mathbf{0 1}) \\
\mathbf{K}_{\mathbf{s}}^{\text {in }} & \begin{array}{l}
\text { Intra community degree } \\
\text { of community }
\end{array} \\
\mathbf{K}_{\mathbf{s}} & \begin{array}{l}
\text { Total community degree } \\
\mathbf{M}
\end{array} \\
& \begin{array}{l}
\text { Total no of edge in } \\
\text { network }
\end{array}
\end{array}
$$

Shen et al [07] proposed extension of modularity to evaluate the quality of overlapped community detection.

$$
\mathbf{E Q}=\sum_{\mathbf{i}} \sum_{\mathbf{w} \in \mathrm{Ci}} \frac{1}{0 v * 0 \mathrm{w}}\left[\mathbf{A}_{\mathrm{vw}}-\frac{\mathrm{K}_{\mathrm{V} * \mathrm{~K}_{\mathrm{w}}}}{2 \mathrm{M}}\right] . .(02)
$$

$\mathrm{M} \quad$ Number of edge in social network

$\mathrm{C}_{\mathrm{i}} \quad$ ith community

Ov Number of community to which the node $\mathrm{v}$ belongs

Avw $=1$ If an edge between node $\mathrm{v}$ and $\mathrm{w}$

$\mathrm{Kv} \quad$ Degree of node $\mathrm{v}$

\subsubsection{Dynamic Modularity}

Takaffoli et al [09] proposed a dynamic modularity to find out detection in dynamic network.

$$
\mathbf{D Q} \mathbf{Q}_{\mathbf{i}}=\boldsymbol{\alpha} \mathbf{Q}\left(\mathbf{G}_{\mathbf{i}}, \mathbf{C}_{\mathbf{i}}\right)+(\mathbf{1}-\boldsymbol{\alpha}) * \mathbf{Q}\left(\mathbf{G}_{\mathbf{i}-\mathbf{1}}, \mathbf{C}_{\mathbf{i}}\right)
$$

$$
\begin{gathered}
\left(\mathrm{G}_{\mathrm{i}}, \mathrm{C}_{\mathrm{i}}\right) \quad \begin{array}{c}
\text { Modularity with respect to partition } \mathrm{C} \\
\text { at time step i. }
\end{array} \\
\left(\mathrm{G}_{\mathrm{i}-1}, \mathrm{C}_{\mathrm{i}}\right) \quad \begin{array}{c}
\text { Modularity of } \mathrm{G} \text { at time }(\mathrm{i}-1), \\
\text { with respect to } \mathrm{C} .
\end{array}
\end{gathered}
$$

\section{PROPOSED METHOD}

In this paper, we purpose our model and explain in detail at each time step.

\subsection{Frame work of proposed model}

At every time step, a dynamic social network may show node and edge changes. Modularity [06] has been proposed to assess the quality of community partition. When the modularity and the community partition at each time step are given.

\section{Proposed Algorithm}

Social network

Modularity of $\mathrm{G}$

Expected modularity

Max iteration

Threshold
G

Q'

Q

Flip Count

$\theta$
Step1 Pick one network from series of network.

Step2 Find modularity of network and store in (Q').

Step3 Real network has modularity range (0.3-0.7) and we take a value as expected modularity in $(\mathrm{Q})$.

\section{Step4 if $\left[\left(Q^{-} Q^{\prime}<=0.1\right)\right]$ \\ Return G', Q' \\ else:}

Step5 We apply node evolution.

Step6 (i) select two nodes.

I Move a clique from one community to other community.

II Again calculate modularity and store in ( tempQ ).

Step7 Again check limiting condition if [(tempQ-Q'<=0.1)]

Return G,Q'

else:

Step8 we apply edge evolution by flip function and calculate modularity.

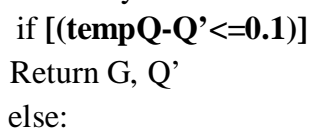

Step9 Apply acceptance probability. 


$$
\mathbf{P a}(\mathbf{t})=\boldsymbol{\alpha} \mathbf{e}^{-\left(\frac{\beta \mathbf{t}}{\mathrm{T}}\right)}
$$

Step10 Find the value of probability and repeat step 5.

Step11 Program stop.

if (iteration = flip count).

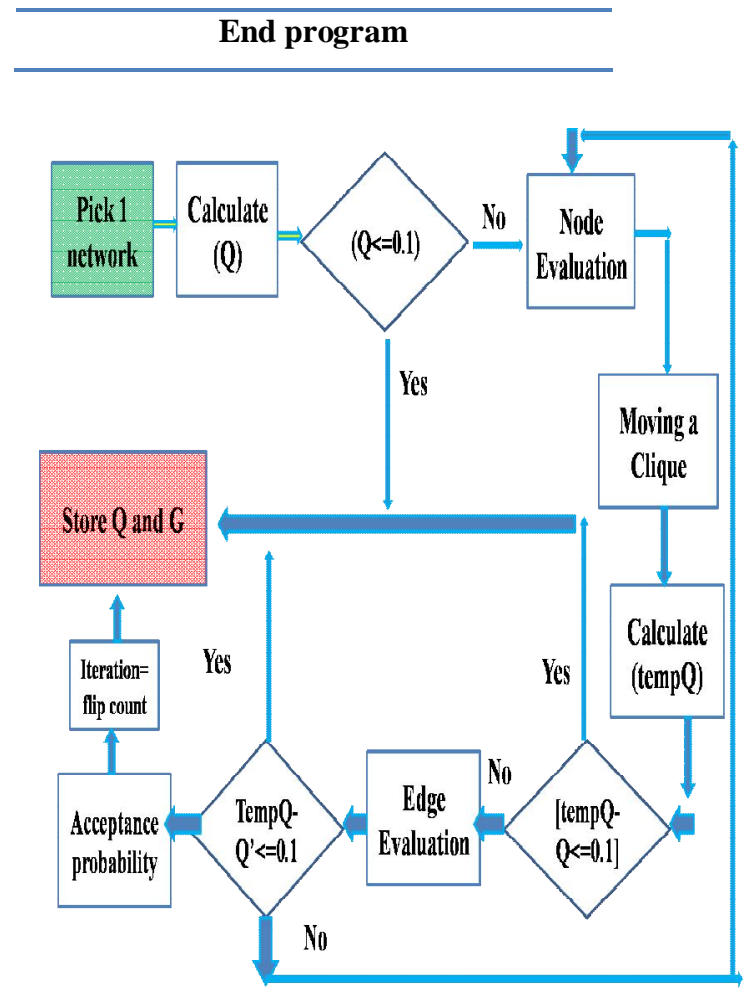

Figure 1 Flow Chart of Proposed Work

. In algorithm, step1 is take a dataset of network and in step2 find out modularity of network and store in (Q') .Step3, In case of real network modularity lies in (0.3-0.7). We take a random value for initialize the program. In step4 we check that the difference between (Q-Q') is satisfy then network modularity is final modularity and execution stop. In Step5, 6, we apply node evolution in node evolution we move a clique change two node from one Community to other community to with ceil function and probability Step7 now again check the threshold condition if satisfy then stop otherwise In step8 we apply edge evolution with the help of flip function in which pick two nodes if there is an edge then delete it or if there is no edge then insert a edge after doing this we again calculate modularity.

Again check threshold condition if condition hold, then execution is stop. In step9 we go acceptance probability.

$$
\operatorname{Pa}(t)=\alpha e^{-\left(\frac{\beta t}{T}\right)}
$$

Where $\alpha=1$, $\mathrm{t}=$ current iteration, $\beta=\operatorname{sign}\left[\mid Q^{\prime}-\right.$ temp $Q \mid-$ $\left.\left|Q^{\prime}-Q\right|\right]=1$

This acceptance probability [10] is inspired by annealing theorem. In step10 take $\mathrm{P}_{\mathrm{o}}$ and repeat step5 and in step11 when no of iteration goes to max count then network store and modularity is store in $\mathrm{Q}$.

\section{EXPERMENTAL RESULT AND ANALYSIS}

\subsection{Software implementation}

In this paper, we use given software.

i. IPYTHON 7.1 This software is used to run the program.

\section{ii. Dynamic Network Generator}

With the help of this software we study network and other parameter of network.

\subsection{Real network}

In the proposed model, we apply two data sets and detail of both data sets is given below.

Table 1 Original Social Network

\begin{tabular}{|c|c|c|}
\hline Name & Node & Community \\
\hline Football & 115 & 12 \\
\hline polbooks & 105 & 03 \\
\hline
\end{tabular}

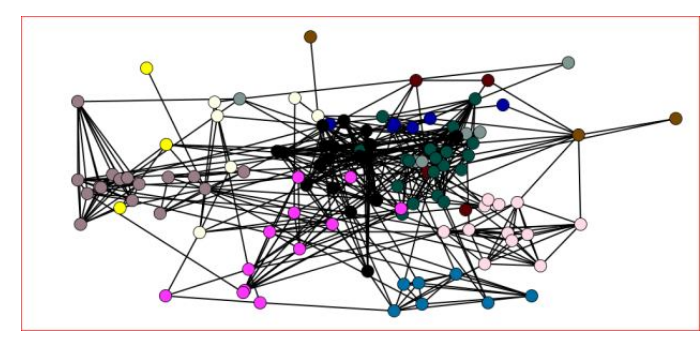

Figure 2 Football network.

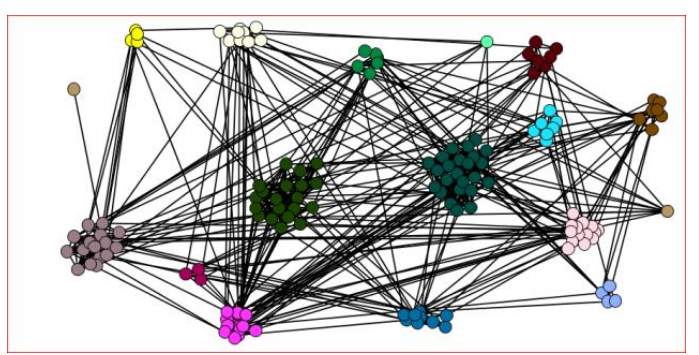

Figure 3 Detected network of football after 10 Iteration. 


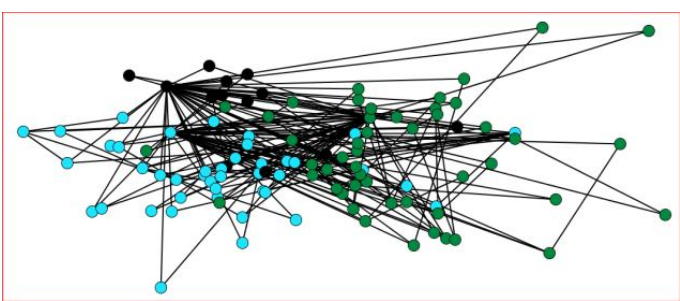

Figure 4 Polbooks network

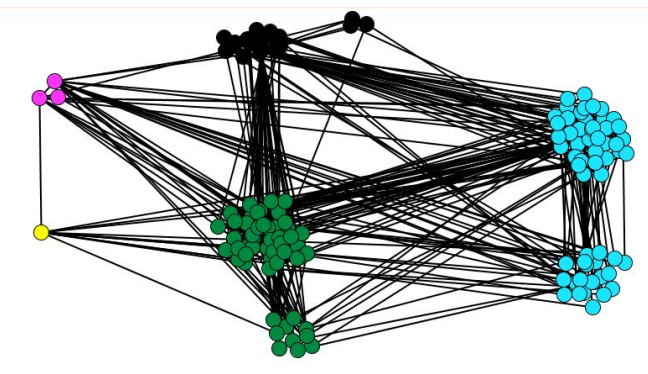

Figure 5 Detected networks of polbooks after 10 Iteration.

In football network different color shows different community and 12 communities. In polbooks network, contain 3 communities.

\subsection{Analysis of network characteristics}

\subsubsection{Modularity}

Modularity value on dynamic network and proposed method are listed in table.

Table 2 Modularity for football network different method

\begin{tabular}{|l|l|l|l|}
\hline \multirow{2}{*}{ Method } & \multicolumn{2}{|c|}{ Change in Modularity } & \multirow{2}{*}{ Change } \\
\cline { 2 - 3 } & $\begin{array}{l}\text { Initail } \\
\text { value }\left(\mathrm{t}_{\mathrm{o}}\right)\end{array}$ & $\begin{array}{l}\text { Final } \\
\text { value }\left(\mathrm{t}_{10}\right)\end{array}$ & \\
\hline TESN-M & 0.59 & 0.30 & $\begin{array}{l}-0.29 \\
\text { (decrease) }\end{array}$ \\
\hline LPA & 0.58 & 0 & $\begin{array}{l}-0.58 \\
\text { (decrease) }\end{array}$ \\
\hline Muktilevel & 0.57 & 0.21 & $\begin{array}{l}-0.36 \\
\text { (decrease) }\end{array}$ \\
\hline CDFR & 0.58 & 0.31 & $\begin{array}{l}-0.27 \\
\text { (decrease) }\end{array}$ \\
\hline $\begin{array}{l}\text { Proposed } \\
\text { Method }\end{array}$ & $\mathbf{0 . 3 6}$ & $\mathbf{0 . 4 9}$ & $\begin{array}{l}\mathbf{+ 0 . 1 3} \\
\text { (Increase) }\end{array}$ \\
\hline
\end{tabular}

Graph of modularity with respect to time for football network is given below.

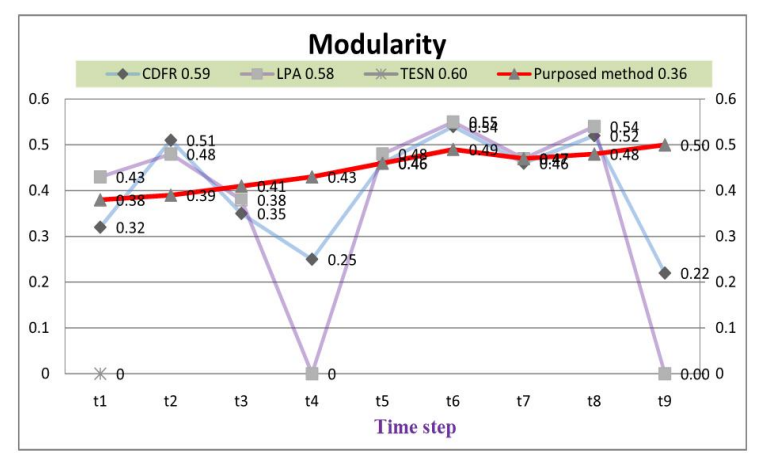

Figure 6 modularity vs iteration.

\subsubsection{Nodes}

Figure6 showes that number of inserted node in dynamic network is increase in proposed method as compare to TESN-M(Time evolving of social netwrok based on modularity).

Table 3 Football network

\begin{tabular}{|c|c|c|}
\hline method & Football & Polbooks \\
\hline & Inserted node & Inserted node \\
\hline TESN-M & 2 & 4 \\
\hline $\begin{array}{c}\text { Proposed } \\
\text { Method }\end{array}$ & $\mathbf{4 0}$ & $\mathbf{2 2}$ \\
\hline
\end{tabular}

Graph of inserted node for both network is given below.

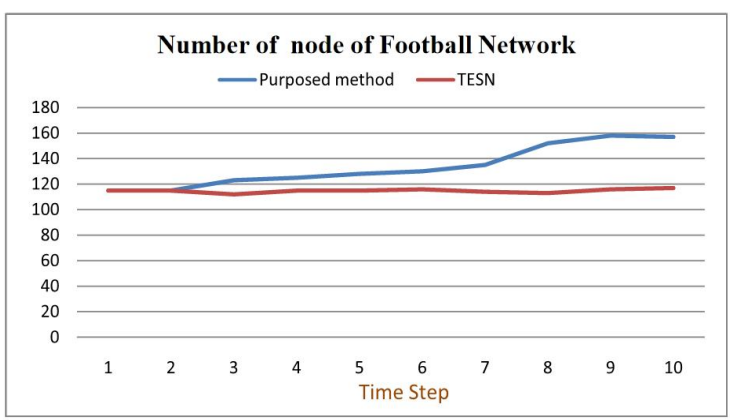

Figure 7 Inserted node vs time step for football network

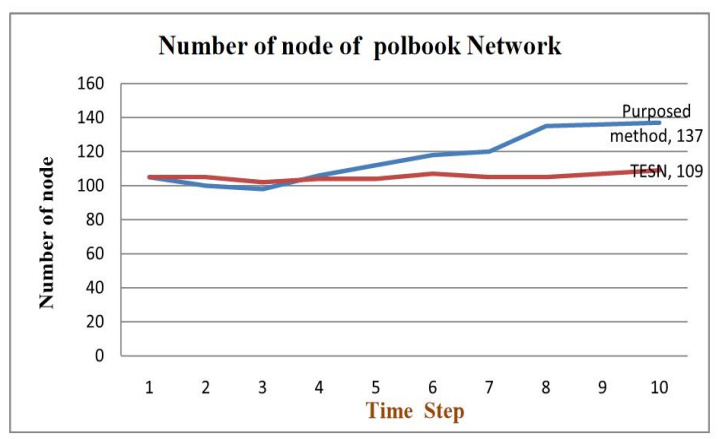

Figure 8 Inserted node vs time step for polbooks network 


\subsubsection{Clustering coffiecient}

Clustering coffiecient reprsent the probabilty that neighbours of nodes are neighbour of each other. Clustering coefficient is a calculate of the degree to which nodes in a network tend to cluster together.

Clustering coefficient of proposed method and TESN are given below.

Table4 C.C. for football and polbook network

\begin{tabular}{|c|c|c|c|}
\hline \multirow[t]{2}{*}{ Method } & \multicolumn{2}{|c|}{$\begin{array}{c}\text { Change in Clustering } \\
\text { coffieicient }\end{array}$} & \multirow[t]{2}{*}{ Change } \\
\hline & $\begin{array}{l}\text { Initail } \\
\text { value }\left(\mathrm{t}_{\mathrm{o}}\right)\end{array}$ & $\begin{array}{l}\text { Final } \\
\text { value }\left(\mathrm{t}_{10}\right)\end{array}$ & \\
\hline \multicolumn{4}{|c|}{ Football network } \\
\hline TESN-M & 0.400 & 0.096 & $\begin{array}{l}-0.304 \\
\text { (decrease) }\end{array}$ \\
\hline $\begin{array}{l}\text { Proposed } \\
\text { method }\end{array}$ & 0.025 & 0.150 & $\begin{array}{l}+1.250 \\
\text { (Increase) }\end{array}$ \\
\hline \multicolumn{4}{|c|}{ Polbook network } \\
\hline TESN-M & 0.48 & 0.11 & $\begin{array}{l}-0.378 \\
\text { (decrease) }\end{array}$ \\
\hline $\begin{array}{l}\text { Proposed } \\
\text { method }\end{array}$ & 0.30 & 0.15 & $\begin{array}{l}-\mathbf{- 0 . 1 5} \\
\text { (decrease) }\end{array}$ \\
\hline
\end{tabular}

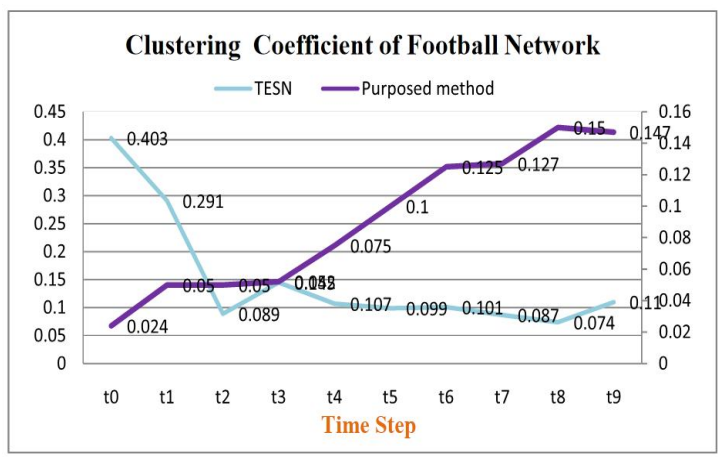

Figure 9 C.C. for football network

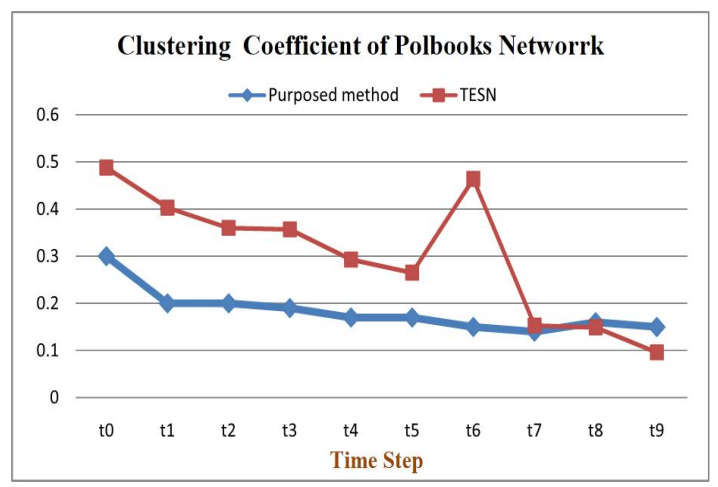

Figure 10 C.C. for polbook network.

\section{CONCLUSION}

In this paper, we have proposed method for dynamic social network. This method shows that modularity in dynamic network detcetion is increase while in other method it decrease for every time step. Other parameter of network like number of inserted node and clustering cofficient are increase.

In the future, we will focus on time evolving social network generators which involve complex structural changes

\section{REFERENCES}

1. Wenjien Luo,Binyo Duan,Li Ni ,'Time evolving social network generator based on modularity," IEEE Transctions on computational social system , 2020.

2. B. Duan, W. Luo, H. Jiang, and L. Ni, "Dynamic social networks generator based on modularity: DSNG-M," in Proc. 2nd Int. Conf. Data Intell. Secur. (ICDIS), Jun. 2019, pp. 167-173.

3. W. Luo, Z. Yan, C. Bu, and D. Zhang, "Community detection by fuzzy relations," IEEE Trans. Emerg. Topics Comput., to be published, doi: 10.1109/TETC.2017.2751101.

4. R. Shang, W. Zhang, L. Jiao, R. Stolkin, and Y. Xue, "A community integration strategy based on an improved modularity density increment for largescale networks," Phys. A, Stat. Mech. Appl., vol. 469,pp. 471-485, Mar. 2017.

5. J. Wu et al., "Overlapping community detection via network dynamics," Phys. Rev. E, Stat. Phys. Plasmas Fluids Relat. Interdiscip. Top., vol. 85,no. 1, Jan. 2012, Art. no. 016115.

6. M. E. J. Newman, "Modularity and community structure in networks," Proc. Nat. Acad. Sci. USA, vol. 103, no. 23, pp. 8577-8582, Jun. 2006.

7. H. Shen, X. Cheng, K. Cai, and M.-B. Hu, "Detect overlapping and hierarchical community structure in networks," Phys. A, Stat. Mech. Appl., vol. 388, no. 8, pp. 1706-1712, Apr. 2009.

8. O. Benyahia, C. Largeron, B. Jeudy, and O. R. Zaïane, "DANCEer: Dynamic attributed network with community structure generator," in Proc. Joint Eur. Conf. Mach. Learn. Knowl. Discovery Databases. Cham, Switzerland: Springer, 2016, pp. 41-44.

9. M. Takaffoli, R. Rabbany, and O. R. Zaïane, "Incremental local community identification in dynamic social networks," in Proc. IEEE/ACM Int. Conf. Adv. Social Netw. Anal. Mining (ASONAM), Aug. 2013, pp. 90-94. 
10. S. Kirkpatrick, C. D. Gelatt, and M. P. Vecchi, "Optimization by simulated annealing," Science, vol. 220, no. 4598, pp. 671-680, 1983.

11. A. Clauset, M. E. J. Newman, and C. Moore, "Finding community structure in very large networks," Phys. Rev. E, Stat. Phys. Plasmas Fluids Relat. Interdiscip. Top., vol. 70, no. 6, Dec. 2004, Art. no. 066111.

12. M. E. J. Newman and M. Girvan, "Finding and evaluating community structure in networks," Phys. Rev. E, Stat. Phys. Plasmas Fluids Relat. Interdiscip. Top., vol. 69, no. 2, Feb. 2004, Art. no. 026113.

13. Aktunc R. et. al., "A dynamic modularity based community detection algorithm for large-scale networks: DSLM", 2015 IEEE/ACM International Conference on Advances in Social Networks Analysis and Mining (ASONAM).

14. Dr. S.K. Chaya Devi, Varsha Reddy Kumbham, Devika Boddu,' A Survey on Databases and Algorithms used for Speech Emotion Recognition', p.p.(7031 -7039), in 2020, doi.org/10.30534/ijatcse/2020/23952020, in ijatcse journal. 